\title{
Stable and unstable variant manifolds in a partially chaotic magnetic configuration generated by nonlinear reconnection
}

Citation for published version (APA):

Borgogno, D., Grasso, D., Pegoraro, F., \& Schep, T. J. (2008). Stable and unstable variant manifolds in a partially chaotic magnetic configuration generated by nonlinear reconnection. Physics of Plasmas, 15(10), 102308-1/7. https://doi.org/10.1063/1.2999539

DOI:

10.1063/1.2999539

Document status and date:

Published: 01/01/2008

Document Version:

Publisher's PDF, also known as Version of Record (includes final page, issue and volume numbers)

Please check the document version of this publication:

- A submitted manuscript is the version of the article upon submission and before peer-review. There can be important differences between the submitted version and the official published version of record. People interested in the research are advised to contact the author for the final version of the publication, or visit the $\mathrm{DOI}$ to the publisher's website.

- The final author version and the galley proof are versions of the publication after peer review.

- The final published version features the final layout of the paper including the volume, issue and page numbers.

Link to publication

\section{General rights}

Copyright and moral rights for the publications made accessible in the public portal are retained by the authors and/or other copyright owners and it is a condition of accessing publications that users recognise and abide by the legal requirements associated with these rights.

- Users may download and print one copy of any publication from the public portal for the purpose of private study or research.

- You may not further distribute the material or use it for any profit-making activity or commercial gain

- You may freely distribute the URL identifying the publication in the public portal.

If the publication is distributed under the terms of Article 25fa of the Dutch Copyright Act, indicated by the "Taverne" license above, please follow below link for the End User Agreement:

www.tue.nl/taverne

Take down policy

If you believe that this document breaches copyright please contact us at:

openaccess@tue.nl

providing details and we will investigate your claim. 


\title{
Stable and unstable invariant manifolds in a partially chaotic magnetic configuration generated by nonlinear reconnection
}

\author{
D. Borgogno, ${ }^{1}$ D. Grasso, ${ }^{2,3}$ F. Pegoraro, ${ }^{4}$ and T. J. Schep ${ }^{5}$ \\ ${ }^{1}$ Université de Nice-Sophia Antipolis, CNRS, OCA, Nice, France \\ ${ }^{2}$ Burning Plasma Research Group, Dipartamento di Energetica, Politecnico di Torino, Torino, Italy \\ ${ }^{3}$ CNR-INFM, Genova, Italy \\ ${ }^{4}$ Physics Department, Pisa University, Pisa, CNISM, Italy \\ ${ }^{5}$ Physics Department, Eindhoven University of Technology, Eindhoven, The Netherlands
}

(Received 5 June 2008; accepted 23 September 2008; published online 22 October 2008)

\begin{abstract}
A numerical contour dynamics code has been employed to calculate the stable and unstable manifolds related to two interacting magnetic island chains. The magnetic configuration is generated by a nonlinear reconnection process described in D. Borgogno et al. [Phys. Plasmas. 12, 032309 (2005)]. The appearance of the first homoclinic and heteroclinic intersections of the dominant manifolds are shown and one of the associated uniformly hyperbolic orbits is given. The stickiness of the field lines around the island and the eventual development of global stochasticity are discussed. The basic geometry of the magnetic configuration is periodic so that the structure of the manifolds may be compared with the one obtained with Poincaré plots. () 2008 American Institute of Physics. [DOI: 10.1063/1.2999539]
\end{abstract}

\section{INTRODUCTION}

The analysis of stochastic fields relies heavily on the use of mapping techniques and on the method of Poincaré plots. Examples are magnetic field line tracing in toroidal plasmas ${ }^{1}$ and flow analysis in oscillating fluids. ${ }^{2}$ Hyperbolic fixed points and the associated stable and unstable manifolds play an essential role in such an analysis of periodic systems. One of the first discussions of the topology of stable and unstable manifolds in toroidal plasmas is presented in Ref. 3. None of these techniques, however, can be used in systems with aperiodic time behavior. In this paper we use the numerical methods of contour dynamics (CD) (Ref. 4) to obtain the invariant manifolds associated with the dominant hyperbolic points. Although CD originated in the area of plasma physics, the numerical method came to full maturity in fluid dynamics. ${ }^{5,6}$ This method is not restricted to periodic systems, and, we will look at its results from the point of view of recent theories ${ }^{7-9}$ on the generalizations of the concepts of hyperbolic points and of their stable and unstable manifolds to general aperiodic systems. These generalizations were discussed within the context of detecting hyperbolic structures in two-dimensional velocity fields and of defining the boundaries of coherent structures.

The system we treat in this paper is a current layer in a sheared magnetic field. The boundary conditions are such that the plasma is unstable and magnetic field line reconnection occurs. During the nonlinear phase of this reconnection process the magnetic field becomes more and more stochastic until the stochastic field region spreads over the whole layer.

In the present paper we will not directly deal with the time evolution of the magnetic field, but we will be interested in the spatial structure of this field at some particular points in time during the reconnection process as described in Ref. 10. This spatial structure is governed by the equations for the magnetic field lines. The magnetic field is represented as

$$
\mathbf{B}=B_{0} \mathbf{e}_{z}+\mathbf{e}_{z} \times \nabla \Psi,
$$

where $B_{0}$ is constant and normalized to unity, and $\Psi=\Psi(x, y, z, t)$ is the poloidal magnetic flux function, which is advanced in time according to the set of dynamical equations given in Ref. 10.

The field lines equations are

$$
\frac{d x}{d z}=-\frac{\partial \Psi}{\partial y}, \quad \frac{d y}{d z}=\frac{\partial \Psi}{\partial x} .
$$

The system is integrable if the flux function does not depend on the axial variable $z$. Then, the elliptic and hyperbolic points of Eq. (2) are solutions for which its right-hand side vanishes, i.e., for which $\mathbf{B}_{*}=\mathbf{e}_{z} \times \nabla \Psi=0$.

At a hyperbolic point (X-point) lines emanate, the separatrices, that form the stable and unstable manifolds associated with the hyperbolic point. The stable (unstable) manifold consists of the set of field lines that converge towards the hyperbolic point for $z \rightarrow+\infty$ (for $z \rightarrow-\infty$ ). These manifolds, curves in the $(x, y)$ plane in the 2D context, are invariant surfaces that separate topologically different types of field lines, i.e., they separate regions with closed field lines from regions with open field lines. Perturbations that are $z$-dependent will destroy the integrability and will break the separatrices of the hyperbolic points.

In the case of an arbitrary $z$ dependence, the position of an X-point, i.e., a point where the gradient of the flux function vanishes, is also $z$-dependent. The path that is traced out by such a point is, however, not a solution of Eq. (2), i.e., it is not a magnetic field line. It can only be a solution if the right-hand side of Eq. (2) does not depend on the "time" $z$. This means that in 2D motions with arbitrary $z$-dependence, the trajectory of the "instantaneous" X-point of the field is 
not a hyperbolic trajectory and no invariant surfaces are related to it! Nevertheless, there exist hyperbolic trajectories in $3 \mathrm{D}$ phase-space $(x, y, z)$ that are the generalizations of the $2 \mathrm{D}$ hyperbolic points. Such a trajectory is a solution of the equations of motion and, thus, a field line. It has the property that all neighboring field lines approach this trajectory exponentially either forward or backward in "time" $z$. It also means that the largest associated Lyapounov exponent is real and positive. Invariant, stable and unstable manifolds are associated with this trajectory, being the generalizations of the $2 \mathrm{D}$ separatrix surfaces. In dynamical system theory, this special hyperbolic orbit is sometimes called a "distinguished hyperbolic trajectory" (DHT) (Ref. 7) or a trajectory of "uniform hyperbolicity." ", 11 In the field of ordinary differential equations, the main characteristic of such a trajectory is that it possesses an "exponential dichotomy.",7,8

The stable and unstable manifolds cannot intersect themselves, but they can intersect each other. The intersections of the unstable and stable manifolds of the same hyperbolic trajectory form homoclinic tangles, while the intersections between a stable (unstable) and an unstable (stable) manifold belonging to different hyperbolic trajectories are called heteroclinic tangles. This is the essence of chaos. The area of a lobe between two successive intersections is conserved in time $z$ for the incompressible magnetic field. In periodic or quasiperiodic systems, one intersection implies an infinite number of intersections.

In the case of arbitrary, aperiodic $z$-dependence, the standard approach to magnetic reconnection and to field stochastization, used in periodic systems, fails, since the method of Poincaré plots cannot be applied. This means that new methods have to be explored. In these methods hyperbolic trajectories with an exponential dichotomy will play a dominant role. The aim of this paper is to investigate the role of the invariant manifolds associated with a hyperbolic trajectory with an exponential dichotomy for a $2 \frac{1}{2} \mathrm{D}$ reconnection problem, where the fields have two spatial components that depend on all three spatial coordinates.

The actual problem under consideration is not fully aperiodic, however, but is still periodic in $z$ so that the Poincaré technique can still be applied. We will take advantage of this, and will use the (numerical) analysis of the spatial structure of the stable and unstable manifolds of several hyperbolic trajectories to obtain insight and to elucidate the development of chaoticity in Poincaré plots.

Although the curve traced out by an instantaneous $\mathrm{X}$-point is not a field line, the position of such a point is often more easily observed in a Poincare plot than the actual hyperbolic line. In the case of weak periodic perturbations, this instantaneous point may still be useful in finding and analyzing the nearby true hyperbolic trajectory and its manifolds, which are hidden in the chaotic region of the flow field.

\section{HYPERBOLICITY}

Since hyperbolicity is a linear notion, we write $\mathbf{x}(z)$ $=\overline{\mathbf{x}}(z)+\xi(z)$, where $\overline{\mathbf{x}}(z)$ does not need to be an exact solution of the system of equations, but must be an approximate so- lution that is close to an exact one. The position with respect to $\bar{x}$ is denoted by $\xi(z)$. The field line equation (2) is rewritten as the sum of a linear and a nonlinear term

$$
\frac{d \xi}{d z}=\mathbf{F}[x(z), z] \xi+\mathbf{g}(z, \xi) .
$$

The matrix $\mathbf{F}$ is given by

$$
\mathbf{F}(z)=\left(\begin{array}{cc}
-\Psi_{x y} & -\Psi_{y y} \\
\Psi_{x x} & \Psi_{x y}
\end{array}\right)_{\mathbf{x}=\overline{\mathbf{x}}(z)}
$$

and

$$
\mathbf{g}(z, \xi)=\mathbf{B}_{*}(\overline{\mathbf{x}}+\xi, z)-\mathbf{F}(z) \xi-\frac{d \overline{\mathbf{x}}}{d z} .
$$

If nonlinear terms in $\xi$ are neglected $\mathbf{g}(z, \xi)$ reduces to $\mathbf{g}(z)$ and is given by

$$
\mathbf{g}(z)=\left(\begin{array}{c}
-\Psi_{\bar{y}}-d \bar{x} / d z \\
\Psi_{\bar{x}}-d \bar{y} / d z
\end{array}\right) .
$$

If $\mathbf{\mathbf { x }}(z)$ is a solution of the original system (Lagrangian linearization), i.e., if $\overline{\mathbf{x}}(z)$ is a field line, then $\mathbf{g}(z)=0$, and if $\overline{\mathbf{x}}$ is independent of $z$ (Eulerian linearization) one has $\mathbf{g}(z)=\left(-\Psi_{\bar{y}}, \Psi_{\bar{x}}\right)$. A mixed case occurs when $\overline{\mathbf{x}}(z)$ is an instantaneous $X$-point or stagnation point, then $\mathbf{g}(z)=(-d \bar{x} / d z,-d \bar{y} / d z)$.

The curve $\mathbf{\mathbf { x }}(z)$ has to be chosen such that it is close to the hyperbolic curve that has to be found. In general this choice of $\overline{\mathbf{x}}(z)$ is not a simple task, but is a basic complication in this theory. The $\overline{\mathbf{x}}(z)$ trajectory must be chosen such that it remains in the region where the value of the determinant of the matrix $\mathbf{F}$ is negative, i.e., in the region where $\mathbf{F}$ has two real eigenvalues of opposite sign. This suggests a simple and global first criterion where to look for hyperbolic trajectories. One expects to find locally hyperbolic trajectories in regions where

$$
-\Psi_{x y}^{2}+\Psi_{x x} \Psi_{y y}<0
$$

over a long stretch in $z$ along the curve $\mathbf{x}(z)$. This condition is equivalent to the Okubo-Weiss criterion ${ }^{12}$ in fluid theory. In addition, the variations along $z$ of the eigenvectors of $\mathbf{F}(z)$ should remain slow as compared with the time scales set by its eigenvalues. ${ }^{9}$ This might be interpreted as requiring that the Eulerian time scales of the problem should be long as compared with the Lagrangian time scales.

Suppose that $\mathbf{\mathbf { x }}(z)$ can be chosen such that the homogeneous and autonomous part of Eq. (3) possesses the property of "exponential dichotomy." Then, under some additional conditions, Eq. (3) has a unique solution $\xi(z)$ such that $\mathbf{x}(z)=\overline{\mathbf{x}}(z)+\xi(z)$ is the sought after hyperbolic trajectory. This is briefly commented upon in the Appendix.

\section{THE RECONNECTION MODEL}

The stable and unstable manifolds of hyperbolic trajectories are calculated numerically during the nonlinear stages of a collisionless, magnetic reconnection process. The dynamics of this process is described in Ref. 10 on the basis of 
a two-fluid plasma description, ${ }^{13}$ valid in the presence of an intense, externally imposed, magnetic guide field. The reconnecting field has only components in the $x-y$ plane but depends on all three spatial coordinates. This model neglects the magnetic field line curvature and takes the axial magnetic field to be constant. The model retains the contribution coming from the electron temperature, through the ion sound Larmor radius $\rho_{s}$, and from electron inertia, through the electron skin depth $d_{e}$. The electron inertia is the mechanism that breaks the frozen-in condition and allows the rearrangement of the magnetic field topology.

The model equations are solved numerically in a 3D slab geometry starting from a static equilibrium configuration with a one-dimensional shear magnetic field.

The spontaneous reconnection process is induced by multiple helicity perturbations with high values of the linear stability parameter $\Delta^{\prime}$.

We will consider a configuration with background toroidal and poloidal magnetic fields that carries initially a resonant mode at each of two neighboring surfaces.

The magnetic flux function consists of an equilibrium part $\psi_{\mathrm{eq}}(x)$ and a wavelike contribution $\psi(x, y, z ; t)$,

$$
\Psi(x, y, z, t)=\psi_{\mathrm{eq}}(x)+\psi(x, y, z ; t),
$$

where $\psi(x, y, z ; t)$ may be written as a sum over equal helicity perturbations

$$
\psi(x, y, z ; t)=\Sigma_{i} \psi_{i}\left(x, k_{y i} y+k_{z i} z, t\right) .
$$

The surfaces $x=x_{s i}$, where the modes are resonant are characterized by $\mathbf{B}_{\mathrm{eq}} \cdot \nabla \Psi=0$, which yields

$$
\frac{d \psi_{\mathrm{eq}}(x)}{d x}=-\frac{\partial \psi_{i} / \partial z}{\partial \psi_{i} / \partial y}=-\frac{k_{z i}}{k_{y i}},
$$

where $k_{y i}=2 \pi m_{i} / L_{y}, k_{z i}=2 \pi n_{i} / L_{z}$ with $L_{y}=4 \pi, L_{z}=32 \pi$.

The numerical simulations were carried out in a tripleperiodic slab starting from an equilibrium configuration with magnetic flux function $\psi_{\mathrm{eq}}=0.19 \cos (x)$. The initial perturbation consists of a contribution $\psi_{1}$ of a mode centered around surface 1 and a contribution $\psi_{2}$ of a mode centered around surface 2 ,

$$
\begin{aligned}
\psi(x, y, z ; t)= & \hat{\psi}_{1}(x, t) \exp \left(i k_{y 1} y+i k_{z 1} z\right) \\
& +\hat{\psi}_{2}(x, t) \exp \left(i k_{y 2} y+i k_{z 2} z\right) .
\end{aligned}
$$

The functions $\hat{\psi}_{1,2}(x, t)$ approximate the analytic solutions of the linearized dynamical equations. The wave numbers $\left(m_{i}, n_{i}\right)$ of the two components of the perturbation are $(1,0)$, for $i=1$, and $(1,1)$, for $i=2$.

In the small amplitude linear phase, when the two helicities evolve independently from each other, each mode induces a magnetic island chain around its resonant surfaces, $x=x_{s i}$. For the case we present here $x_{s 1}=0, \pi$ and $x_{s 2}$ $=0.71, \pi-0.71$. Since resonant surfaces with $x_{s}>\pi / 2$ are simply due to the periodicity of the magnetic equilibrium $\psi_{\mathrm{eq}}$, they will be omitted and we will focus on the magnetic field structure in the reduced interval $-\pi / 2<x<\pi / 2$. The amplitude $\hat{\psi}_{1}$ is of order $10^{-4}$ and is ten times bigger than $\hat{\psi}_{2}$. The system is unstable with respect to these modes. The magnitudes of their linear growth rates are comparable and continue to apply well into the nonlinear regime. During the reconnection process, modes with different helicity and higher order modes of the same helicity are generated. This also implies that new hyperbolic trajectories are created. These modes often have larger growth rates. The relevant nonlinear modes turn out to still have the same two helicities as the initial modes. The time evolution of this system has been reported in Ref. 10. In what follows we will only consider the dominant manifold structure that is associated with the initial modes.

We are interested in analyzing the structure of the magnetic field when a large number of modes has been generated and the magnetic field has developed a chaotic behavior on a substantial part of the volume between the initial resonant surfaces. We consider here subsequent stages of the dynamics of the reconnection process described above, around the Chirikov regime where the transition to global stochasticity occurs, ${ }^{14}$ when the size of the islands becomes such that they overlap. This corresponds in our $z$-dependent model to the generation of heteroclinic points.

Since the system that we consider does not have an arbitrary $z$-dependence, but is periodic, the method of Poincaré plots can still be applied. Poincaré plots are suitable for treating periodical, time-dependent dynamical systems as the magnetic field we consider here. They collect the intersections with the $(x, y)$ plane of a number of magnetic field lines, calculated by the numerical integration of the magnetic field equations, and allow us to visualize the topology of the magnetic field. In addition we use the method of contour dynamics (CD) which can also be applied in case of fully aperiodic $z$-dependencies.

Contour dynamics ${ }^{5,6}$ is used to determine the position where a trajectory of uniform hyperbolicity cuts the $z=0$-plane and to identify coherent structures (i.e., stable and unstable manifolds and the associated hyperbolic trajectories) inside the regions where the magnetic field exhibits a chaotic behavior. Visualization of stable and unstable manifolds assumes a good approximation of the initial position of the hyperbolic trajectories, which is a nontrivial assumption in highly nonlinear systems. In our analysis the manifolds have been obtained by a numerical code ${ }^{4}$ based on the approximation of the contours by a variable number of linear elements. The simulations were performed starting from small circle on a $z=$ constant-plane, centered at the positions of the corresponding X-points as guessed from the Poincare plot. These contours are repeatedly advected forward and backward in $z$ along the field lines of $\mathbf{B}_{*}$, in order to approximate the point where the DHT cuts the $z=$ constant plane and to generate the unstable and stable manifolds, respectively.

\section{NUMERICAL RESULTS}

In this section we compare the magnetic field structures produced by the nonlinear development of the reconnection process at three different times, $t=415 \tau_{A}, t=425 \tau_{A}, t$ $=455 \tau_{A}$, as obtained in the numerical simulations reported in Ref. 10. During this time interval a rapid change occurs in the width of the domain where the magnetic field lines are 

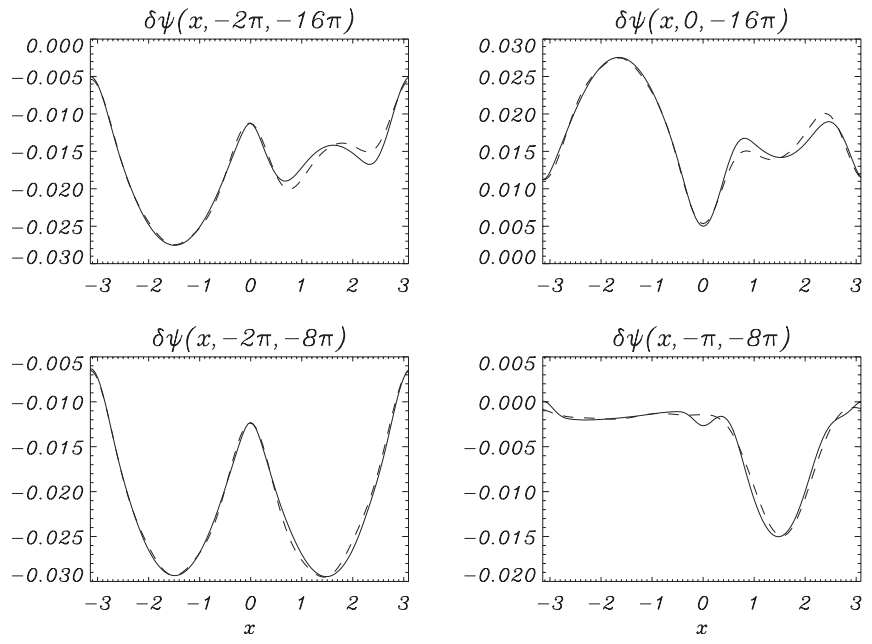

FIG. 1. Comparison between the profiles of the perturbed Hamiltonian $\delta \psi$ $=\psi-\psi_{\text {eq }}$ (solid line), obtained from the simulations, and the adopted approximation (dashed lines) at four different values $(y, z)$ and at $t=455 \tau_{A}$. The chosen approximation contains the 20 largest amplitude components of the Fourier transform of $\delta \psi$.

chaotic, which corresponds to the transition from local to global scale chaoticity. At $t=415 \tau_{A}$ the magnetic field line chaoticity domains are still localized inside thin layers along the separatrices of the two magnetic islands generated by the initial perturbations. These domains grow as the reconnection instability develops and at $t=425 \tau_{A}$ they merge. From this time on a single macroscopic chaotic region is formed.

In order to simplify the computation of the manifolds, that typically appear extremely deformed and folded in the chaotic regions, we will use approximate descriptions of the Hamiltonian function $\psi$. In Fig. 1 we show a comparison between the perturbed Hamiltonian $\delta \psi=\psi-\psi_{\text {eq }}$ (solid line), as obtained from the dynamics described in Ref. 10, and the adopted approximation (dashed line), evaluated at fixed values of $(y, z)$ and at $t=455 \tau_{A}$. Low amplitude modes are suppressed in the approximated flux function which represents 20 modes. For the evolution times considered here it turns out that these latter modes have the helicity of either of the original perturbations.

In the following figures we show the Poincare plots, that identify the regions where the magnetic field lines exhibit a chaotic behavior, together with the manifolds obtained with the CD-code, at subsequent times of the reconnection process as described in Ref. 10 and at different cross sections along $z$. The magnetic field line trajectories are calculated by means of a kinematic code based on a standard variableorder, variable-step Adams method, ${ }^{15}$ which integrates the Hamilton equations (2) over a suitable range of initial conditions, for a given interval along the $z$ direction.

Each point of the manifold can be considered as the initial position of a field line. Since the magnitude and the direction of the magnetic field at neighboring points are almost equal, the small structures that are created by the folding manifolds are not true small scales of the physical problem.

All figures show a partial visualization of the manifolds calculated at $z=n \cdot L_{z}$, where $n$ is a integer number. The rep-

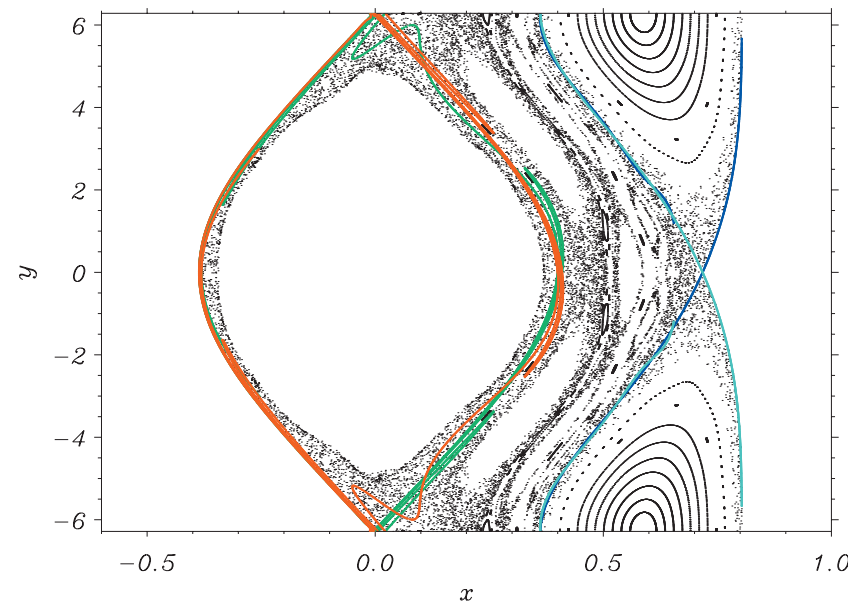

FIG. 2. (Color) Poincaré map of the magnetic field on the section $z=0$ at $t=415 \tau_{A}$. The area filled by black points shows the region where magnetic chaos is confined. The superimposed red and green lines represent the corresponding stable and unstable manifolds calculated by the $\mathrm{CD}$ code with initial conditions centered at $(0,-2 \pi)$, while dark and light blue curves show the manifolds rising at $(0.71,0)$. While the Poincaré map has been obtained integrating the dynamical equations for magnetic field lines up to $z=1000 L_{z}$ and starting with 30 initial conditions distributed at $y=-2 \pi$ between $x=0$ and $x=0.56$, for each manifold the CD integration has been performed until $z=6 L_{z}$.

resentation of the magnetic structure becomes increasingly accurate with the number of iterates $n$.

The manifolds are obtained initializing the CD code with conditions centered around two points $\left(x_{X 1}, y_{X 1}\right)$ (red and green curves) and $\left(x_{X 2}, y_{X 2}\right)$ (dark and light blue curves). At the beginning of the nonlinear phase, when the perturbations are still small, these points are located at $\left(x_{X 1}, y_{X 1}\right)$ $=(0,-2 \pi)$ and $\left(x_{X 2}, y_{X 2}\right)=(0.71,0)$, which correspond to the $\mathrm{X}$-points induced by the small amplitude initial perturbation in Eq. (10) on the plane $z=0$. When the amplitudes of the perturbations grow, the manifolds move, hence the location of $\left(x_{X 1}, y_{X 1}\right)$ and $\left(x_{X 2}, y_{X 2}\right)$ have been changed in order to identify the DHT.

In order to analyze the relation between the manifold structure and the magnetic field topology, each figure contains the corresponding Poincare plot on the $z=0$ plane.

Figure 2 shows the Poincare plot and the manifolds at an early stage of the nonlinear phase of the reconnection process $\left(t=415 \tau_{A}\right)$. At this stage the main modes have grown by two orders of magnitude as compared with their initial values. The CD integration has been continued up to $z=6 L_{z}$. The presence of a couple of hyperbolic points together with the associated stable and unstable manifolds is clearly visible. These points identify the positions where two DHTs cross the $z=$ constant section.

It is seen that the largest homoclinic tangle is associated with the manifold related to the $(1,0)$ mode. If the CD integration is continued, a similar tangle associated with the $(1,1)$ mode will appear, but on a smaller scale. This difference is due to the fact that the amplitude of the magnetic perturbation around $x=x_{X 2}$ is much smaller than in the points surrounding $x=x_{X 1}$ and, as a consequence, the magnetic field is less chaotic. 


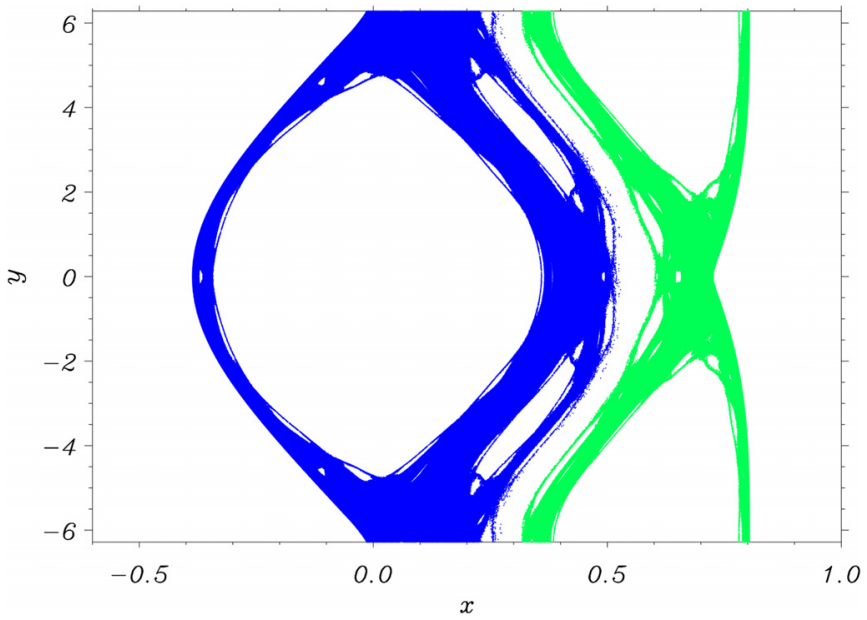

FIG. 3. (Color online) Poincaré plot obtained at $t=415 \tau_{A}$ after 500 iterations, which corresponds to $z=500 L_{z}$, putting 5000 initial conditions around $(0,-2 \pi)$ [black (blue online) dots] and $(0.71,0)$ [gray (green online) dots]. We can appreciate that no heteroclinic intersection has been occurred.

Although the Poincaré plot shows stochastic regions, there is no sign of heteroclinic intersections between the manifolds of the dominant modes. This is also emphasized in Fig. 3, which shows the unstable manifold associated with the $(1,0)$ mode (blue) and the stable manifold associated with the $(1,1)$ mode (green) after $5 \times 10^{2}$ iterations of the field lines, which corresponds to $z=500 L_{z}$. These manifolds have been obtained by the kinematic code, integrating the field line equations, forward and backward in time $z$, respectively. The code has been initialized with 5000 conditions located at the hyperbolic points identified by the $\mathrm{CD}$ code. Heteroclinic intersections do not occur. This means that at this time in the discharge the Poincare plot cannot be produced by following a single field line.

Figures 4 and 5 show the equivalent results at time $t$ $=425 \tau_{A}$. Already after a few turns around the system the CD

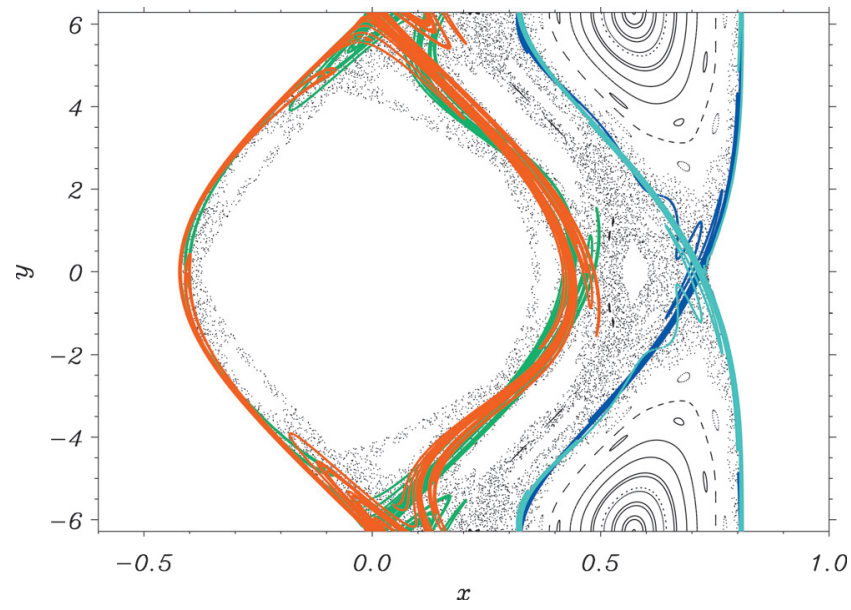

FIG. 4. (Color) Poincaré map of the magnetic field on the section $z=0$ at $t=425 \tau_{A}$, with superimposed corresponding pairs of manifolds rising at $(0,-2 \pi)$ (green and red curves) and $(0.72,0)$ (dark and light blue curves). Each set of manifolds have been calculated up to $z=10 L_{z}$, while the Poincaré map has been obtained integrating the dynamical equations for magnetic field lines up to $z=1000 L_{z}$ and starting with 30 initial conditions distributed at $y=-2 \pi$ between $x=0$ and $x=0.66$.

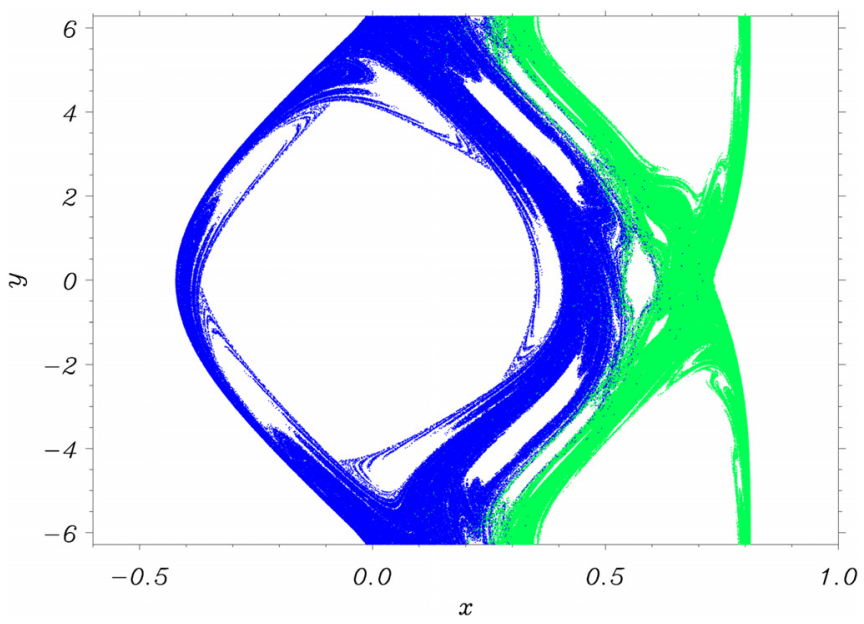

FIG. 5. (Color online) Poincaré plot obtained at $t=425 \tau_{A}$ after 50 iterations, with 10000 initial conditions located around $(0,-2 \pi)$ [black (blue online) dots] and $(0.72,0)$ [gray (green online) dots]. We see that at this stage heteroclinic intersections have already occurred.

code shows the occurrence of homoclinic points of each set of manifolds. The manifolds stick to the island structures for many turns around the system time before wandering off ${ }^{16}$ and heteroclinic points appear only after many iterations around the system.

Figure 6 shows the manifolds at time $t=455 \tau_{A}$. At this time the amplitudes of the two main perturbations have reached the fully nonlinear regime. It is seen that at this time the unstable/stable manifold associated with one hyperbolic trajectory cuts the stable/unstable manifold related to the other hyperbolic line. At that stage the magnetic field of the two island chains is globally stochastic. The generation of higher order island structures influences locally the degree of stochastization, the global stochastization turns out to be due to the entanglement of the stable and unstable manifolds of the dominant modes.

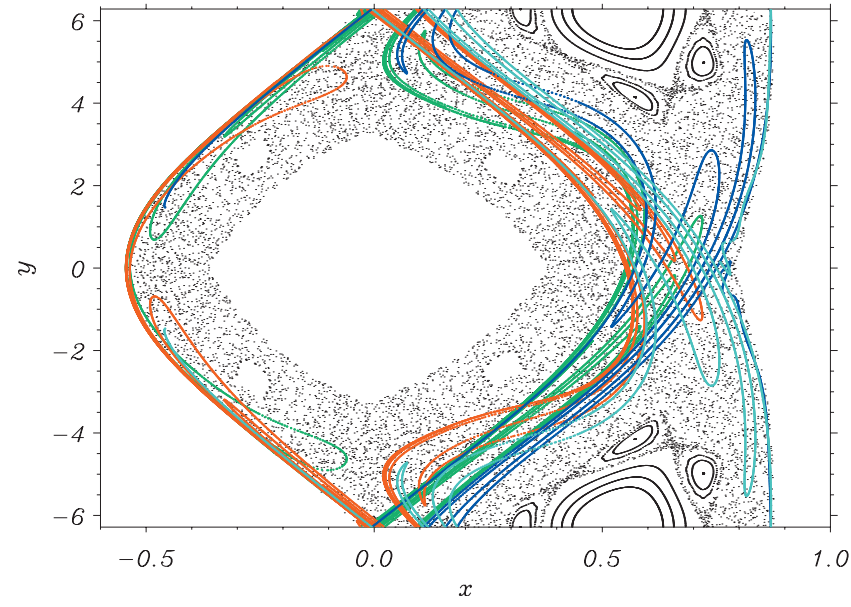

FIG. 6. (Color) Poincaré map of the magnetic field on the section $z=0$ at $t=455 \tau_{A}$, with superimposed the corresponding pairs of manifolds rising at $(0,-2 \pi)$ (green and red curves) and $(0.8,0)$ (dark and light blue curves). The integration of the manifolds has been continued up to $10 L_{z}$, while the Poincaré map has been obtained integrating the dynamical equations for magnetic field lines up to $z=1000 L_{z}$ and starting with 20 initial conditions, distributed at $y=-2 \pi$ between $x=0$ and $x=0.43$. 

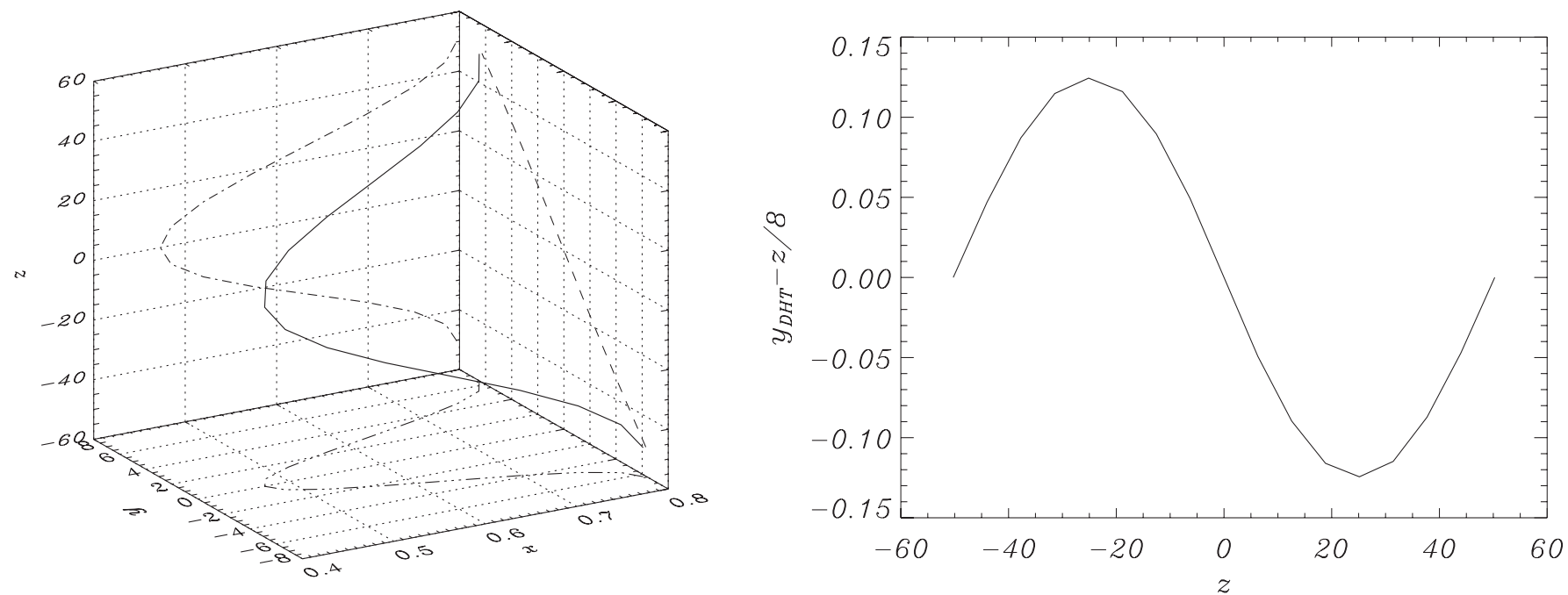

FIG. 7. Left frame: hyperbolic trajectory formed by the cross section of the stable and unstable manifolds associated with the point $\left(x_{X 2}, y_{X 2}\right)$ at $t=455 \tau_{A}$. The projection of the trajectory is also shown on each plane. Right frame: departure of the evolution in $z$ of the DHT $y$-coordinate from the straight line that would represent the evolution of the helicity associated with a pure $(1,1)$ mode.

Looking at the manifolds associated with the $\left(x_{X 1}, y_{X 1}\right)$ point, it is seen that the branches facing the $(1,1)$ mode do not join smoothly but show several homoclinic intersections. This also holds for the branches of the manifolds associated with the $\left(x_{X 2}, y_{X 2}\right)$ point, that face the $(1,0)$ mode. The nonfacing branches on the contrary are much more robust and show either no or a rather small tangle. The facing branches of the two sets tend to intersect and produce heteroclinic points. At this stage the last Kolmogorov-Arnodl-Moser (KAM) surface between the major island structures will have disappeared and one can speak of global stochastization.

The special hyperbolic trajectory that forms the cross section of the stable and unstable manifolds associated with the point $\left(x_{X 2}, y_{X 2}\right)$ is shown in Fig. 7. It is seen that even when the magnetic field is highly chaotic, these points continue to be localized close to the unstable stationary point characteristic of the linear phase of the reconnection instability. This is due the relative small values of the amplitudes. Note that the oscillations in the $x$-direction are approximately half the distance between the two resonant surfaces.

\section{CONCLUSIONS}

Contour dynamics is employed to trace the stable and unstable manifolds associated with hyperbolic points. This $\mathrm{CD}$ method can also be used in aperiodic systems, where the hyperbolic point is generalized to a uniformly hyperbolic line and the manifolds to surfaces in phase space. The main problem will be to guess where the hyperbolic trajectory of interest cuts a $t=$ constant or, in the case of magnetic field lines, a $z=$ constant surface.

A kinematic code, like those used to generate Poincaré plots, is applied to trace the manifolds over longer times or distances.

The different stages of the development of a chaotic magnetic field in a reconnection process have been analyzed. In the first stage (local chaos) only homoclinic intersections occur, while at later times heteroclinic intersections between manifolds belonging to different DHT lead to global chaos.

\section{ACKNOWLEDGMENTS}

The authors would like to acknowledge Dr. R. R. Trieling for providing us the contour dynamics code and useful hints. This work was partly supported by the Euratom Communities under the contract of Association between EURATOM/ENEA. The views and opinions expressed herein do not necessarily reflect those of the European Commission. This work was also partly supported by Grant No. PRIN-2006.

\section{APPENDIX: EXPONENTIAL DICHOTOMY}

In this appendix we briefly describe the property of exponential dichotomy and provide a formal solution of Eq. (3) that can be identified with the DHT.

Let $\mathbf{X}\left(z, z_{0}\right)$ be the matrix that solves the linear autonomous version of Eq. (3), so that

$$
\xi(z)=\mathbf{X}\left(z, z_{0}\right) \xi\left(z_{0}\right), \quad \frac{d \mathbf{X}\left(z, z_{0}\right)}{d z}=\mathbf{F}(z) \mathbf{X}\left(z, z_{0}\right),
$$

$z_{0}$ is the initial time. Then, Eq. (3) is said to possess an exponential dichotomy if projection operators $\mathcal{P}$ and $\mathcal{Q}=\mathcal{I}-\mathcal{P}$ can be found such that

$$
\begin{array}{ll}
\mathbf{X}\left(z, z_{0}\right) \mathcal{P} \mathbf{X}\left(z, z_{0}\right)^{-1} \leqslant K \exp [-\alpha(z-s)] & \text { for } z \geqslant s, \\
\mathbf{X}\left(z, z_{0}\right) \mathcal{Q} \mathbf{X}\left(z, z_{0}\right)^{-1} \leqslant L \exp [-\beta(z-s)] & \text { for } z \leqslant s,
\end{array}
$$

with $(z, s) \epsilon\left[z_{0}, z_{L}\right]$ and $z_{L}$ the final time. The projection operators $P$ and $Q$ project on the stable and on the unstable direction, respectively, and $K, L, \alpha, \beta$ are real positive constants. The above property is used in the proof that the following particular integral solution of Eq. (3) has a unique solution 


$$
\begin{aligned}
\xi(z)= & \mathbf{X}\left(z, z_{0}\right) \int_{-\infty}^{z} \mathcal{P} \mathbf{X}^{-1}\left(s, z_{0}\right) \mathbf{g}[\xi(z), s] d s \\
& -\mathbf{X}\left(z, z_{0}\right) \int_{z}^{+\infty} \mathcal{Q} \mathbf{X}^{-1}\left(s, z_{0}\right) \mathbf{g}[\xi(z), s] d s,
\end{aligned}
$$

where $\mathbf{g}[\xi(z), s]$ is given by Eq. (5). This integral equation has a unique solution if for small $\|\xi\|$ and $\left\|\xi_{1}-\xi_{2}\right\|$,

$$
\begin{aligned}
& \eta\|\mathbf{g}[\xi(z), z]\| \leqslant\|\xi(z)\|, \\
& \eta\left\|\mathbf{g}\left[\xi_{1}(z), z\right]-\mathbf{g}\left[\xi_{2}(z), z\right]\right\| \leqslant \|\left[\xi_{1}(z)-\xi_{2}(z) \|,\right.
\end{aligned}
$$

where $\eta=K / \alpha+L / \beta$, and the norm $\|\ldots\|$ is defined as the maximum value of its argument over $x, y$, and $z$.

Note that the Lyapounov exponent is given in terms of the matrix $\mathbf{X}$ by

$$
\gamma=\frac{1}{2} \lim \frac{1}{\left|z_{L}-z_{0}\right|}\left|\mathbf{X}\left(z_{L}, z_{0}\right) \mathbf{X}^{T}\left(z_{L}, z_{0}\right)\right|,
$$

where the superscript $T$ indicates the transposed matrix.

In some cases, the curve traced out by an instantaneous $\mathrm{X}$-point of the field might be used as an indication of the position of the actual hyperbolic trajectory. However, it should be kept in mind that such X-points are dependent on the choice of the field $\mathbf{B}_{*}$, while the hyperbolic trajectories, being solutions of the full dynamical system, are independent of this choice. The same problem arises when this method is applied to fluid systems in which case the position of the stagnation points of the velocity field is frame dependent as it can be shifted with a Galileo transformation. ${ }^{2}$

If $\overline{\mathbf{x}}(z)$ represents the position of an isolated instantaneous X-point of the field, i.e., a null of the local shear field $\mathbf{B}_{*}$, its "velocity" $\mathbf{v}_{* n}=(d \bar{x} / d z, d \bar{y} / d z)$ can be calculated as follows:

$$
\frac{\partial \mathbf{B}_{*}}{\partial z}+\mathbf{v}_{* n} \cdot \nabla \mathbf{B}_{*}=0, \quad \mathbf{B}_{*}=0 .
$$

Upon using Eq. (2) and inverting the matrix, one obtains the velocity of the magnetic null $\left(\Psi_{\bar{x}}=0, \Psi_{\bar{y}}=0\right)$,

$$
\begin{aligned}
\left(\begin{array}{c}
d \bar{x} / d z \\
d \bar{y} / d z
\end{array}\right) & =-\mathbf{F}^{-1}\left(\begin{array}{c}
-\Psi_{\bar{y} z} \\
\Psi_{\bar{x} z}
\end{array}\right) \\
& =\frac{\mathbf{F}}{F}\left(\begin{array}{c}
-\Psi_{\bar{y} z} \\
\Psi_{\bar{x} z}
\end{array}\right) \\
& =\frac{1}{F}\left(\begin{array}{c}
\Psi_{\overline{x y}} \Psi_{\bar{y} z}-\Psi_{\bar{y} \bar{y}} \Psi_{\bar{x} z} \\
-\Psi_{\overline{x x}} \Psi_{\bar{y} z}+\Psi_{\overline{x y}} \Psi_{\bar{x} z},
\end{array}\right),
\end{aligned}
$$

where $F=\operatorname{det} \mathbf{F}=\Psi_{x x} \Psi_{y y}-\Psi_{x y}^{2}$. In this case, the linearized version of the field line equation (3) becomes

$$
\frac{d \xi}{d z}=\mathbf{F}(z) \xi-\frac{1}{F} \mathbf{F}(z)\left(\begin{array}{c}
-\Psi_{z \bar{y}} \\
\Psi_{z \bar{x}} .
\end{array}\right)
$$

It is seen that the velocity of a null vanishes if the field consists of single helicity modes belonging to the same rational surface as the null $(\partial / \partial z=0)$. If the field consists of single helicity modes belonging to a different mode rational surface, then $\partial / \partial z=\left(k_{z i} / k_{y i}\right) \partial / \partial \bar{y}$ and the null moves along the $y$-axis with the velocity $d \bar{y} / d z=-k_{z i} / k_{y i}$. In case of a mixture of modes belonging to several rational surfaces, the leading order "velocity" of the null in the $y$-direction is,

$$
\frac{d \bar{y}}{d z}=-\frac{\sum_{i}\left(k_{z i} / k_{y i}\right) \psi_{i y y}}{\sum_{i} \psi_{i y y}}
$$

The velocity in the $x$-direction is second order in the mode amplitudes.

\footnotetext{
${ }^{1}$ A. Wingen, M. Jakubowski, K. H. Spatschek, S. S. Abdullaev, K. H. Finken, and M. Lehnen, Phys. Plasmas 14, 042502 (2007).

${ }^{2}$ Y. Ohsugi and M. Funakoshi, Fluid Dyn. Res. 26, 289 (2000).

${ }^{3}$ T. E. Evans, R. K. W. Roeder, J. A. Carter, and B. I. Rapoport, Contrib. Plasma Phys. 44, 235 (2004).

${ }^{4}$ J. H. Mentink, J. Bergmans, L. P. J. Kamp, and T. J. Schep, Phys. Plasmas 12, 052311 (2005).

${ }^{5}$ D. G. Dritschel, Comput. Phys. Rep. 10, 77 (1989).

${ }^{6}$ P. W. C. Vosbeek and R. M. M. Mattheij, J. Comput. Phys. 133, 222 (1997).

${ }^{7}$ K. Ide, D. Small, and S. Wiggins, Nonlinear Processes Geophys. 9, 237 (2002).

${ }^{8}$ N. Ju, D. Small, and S. Wiggins, Int. J. Bifurcation Chaos Appl. Sci. Eng. 13, 1449 (2003).

${ }^{9}$ G. Haller, Chaos Near Resonance (Springer-Verlag, New York, 1999).

${ }^{10}$ D. Borgogno, D. Grasso, F. Califano, F. Farina, F. Pegoraro, and F. Porcelli, Phys. Plasmas 12, 032309 (2005).

${ }^{11}$ Ya. B. Pesin, in Dynamical Systems II, edited by Ya. G. Sinai (Springer Verlag, Berlin, 1989), Chap. 7.

${ }^{12}$ G. Boffeta, G. Lacorata, G. Radelli, and A. Vulpiani, Physica D 159, 58 (2001).

${ }^{13}$ T. J. Schep, F. Pegoraro, and B. N. Kuvshinov, Phys. Plasmas 1, 2843 (1994).

${ }^{14}$ B. V. Chirikov, Phys. Rep. 52, 256 (1979).

${ }^{15}$ www.nag.co.uk/numeric/fl/manual/pdf/D02/d02cjf.pdf.

${ }^{16}$ G. Zaslavsky, Hamiltonian Chaos and Fractional Dynamics (Oxford Uni-
} versity Press, New York, 2005). 